\title{
Geometry or Not Geometry? Perceived Orientation and Spatial Layout in Pictures Viewed at an Angle
}

\author{
E. Bruce Goldstein \\ University of Pittsburgh
}

\begin{abstract}
Cutting (1988) suggests that changes in the perceived orientations of pictured objects that occur with changes in viewing angle are caused by the geometrical changes that accompany these changes in viewing angle. His geometrical analysis does predict the differential rotation effect reported by Goldstein $(1979,1987)$, but fails to predict other important aspects of the data. Cutting's analysis does, however, support Goldstein's (1987) conclusion that in future research on picture perception it is important to clearly distinguish between the attributes of perceived orientation and spatial layout.
\end{abstract}

Cutting's (1988) Observation bears on one of the central questions in picture perception: What is the relation between the geometrical information projected to the eye and the observer's perception of a picture? His analysis, based on $\mathrm{La}$ Gournerie (1859), led him to conclude that perceived orientation, the direction a pictured object appears to point when extended out of the picture, is controlled by geometrical information and that the changes in perceived orientation occuring with changes in viewing angle can, therefore, be explained in terms of the geometrical changes accompanying these changes in viewing angle.

The first step in evaluating Cutting's conclusion is to ask how well the functions predicted by his calculations fit the empirical data. On a general level, the fit between the model and data is good. That is, Cutting's analysis predicts the differential rotation effect (Goldstein, 1979, 1987)—that pictured objects oriented more parallel to the picture plane rotate less in response to an observer's change in viewing angle than do pictured objects that are oriented more perpendicular to the picture plane.

Although Cutting's geometrical model predicts the differential rotation effect, inspection of his Figure 3 shows that the geometrical model leaves some important details unexplained. Although the model fits the data for $B \rightarrow A$ well, the predicted functions for $\mathrm{B} \rightarrow \mathrm{C}$ and $\mathrm{C} \rightarrow \mathrm{A}$ differ appreciably from the data. The horizontal function predicted for $\mathrm{B} \rightarrow \mathrm{C}$ and the slightly curved function predicted for $\mathrm{C} \rightarrow \mathrm{A}$ do not match the sloping functions that are always observed empirically (also see Ellis, Smith, \& McGreevy, in press; Goldstein, 1979). Consider the mismatch between prediction and data for $B \rightarrow C$. The model predicts a constant orientation relative to the picture plane as viewing angle changes-that is, no rotation-but appreciable rotation does, in fact, occur. Thus, although Cutting's model does succeed in duplicating the differential rotation effect, the functions it predicts are different from those determined empirically. Cutting characterizes these differences as a "subsidiary effect," "evident only at

Correspondence concerning this article should be addressed to $\mathrm{E}$. Bruce Goldstein, Department of Psychology, University of Pittsburgh, Pittsburgh, Pennsylvania 15260. extreme slants" (p. 308). But the differences in the forms of these two curves is more than a subsidiary effect, and any model that claims to explain the effects of viewing-at-an-angle should be able to fit the data at viewing angles that deviate appreciably from $90^{\circ}$.

To determine his predicted curves for comparison with the observed results, Cutting used the Rosinski, Mulholland, Degelman, and Farber (1980) equation. Another way to make this comparison is illustrated in Figure 1. This illustration employs graphical techniques (see Cutting, 1986, p. 36; Goldstein, 1987) that have the advantage of making it possible to visualize the predicted and observed orientations. Another advantage of these techniques is that they emphasize an important aspect of Cutting's analysis: His predictions of perceived orientations are based on how affine transformations affect the spatial layouts of objects in a scene. The fact that Cutting's predicted orientations are derived from an analysis of spatial layout raises a theoretically important paradox that I will consider shortly. First, however, I will use the graphical method to compare Cutting's predictions with the empirical results.

The top portion of the figure shows how affine transformations affect the spatial layout defined by Goldstein's (1987) triangular array (see Cutting's Figure 3). The transformations indicate that viewing from $20^{\circ}$ or $160^{\circ}$ causes appreciable distortions in the array. The lower part of the figure shows triangular layouts derived from Goldstein's (1987) empirically determined perceived orientations. The correspondence between the shapes of the upper and lower layouts at $90^{\circ}$ and $160^{\circ}$ mirrors the fit between the predicted and observed data points in Cutting's Figure 3 at these viewing angles. There are, however, two important differences between these two sets of layouts:

1. The empirically determined layouts are oriented differently than the affine transformed layouts. This difference reflects the fact that although $\mathrm{B} \rightarrow \mathrm{C}$ and $\mathrm{C} \rightarrow \mathrm{A}$ exhibit substantial rotation with changes in view, this is not predicted by the geometrical model. Notice how the orientations of lines $\mathrm{BC}$ and CA remain relatively constant in the top triangles but systematically change in the bottom triangles.

2. Although the layouts compare well at the $90^{\circ}$ and $160^{\circ}$ viewing angles, there is a large difference between the layouts 


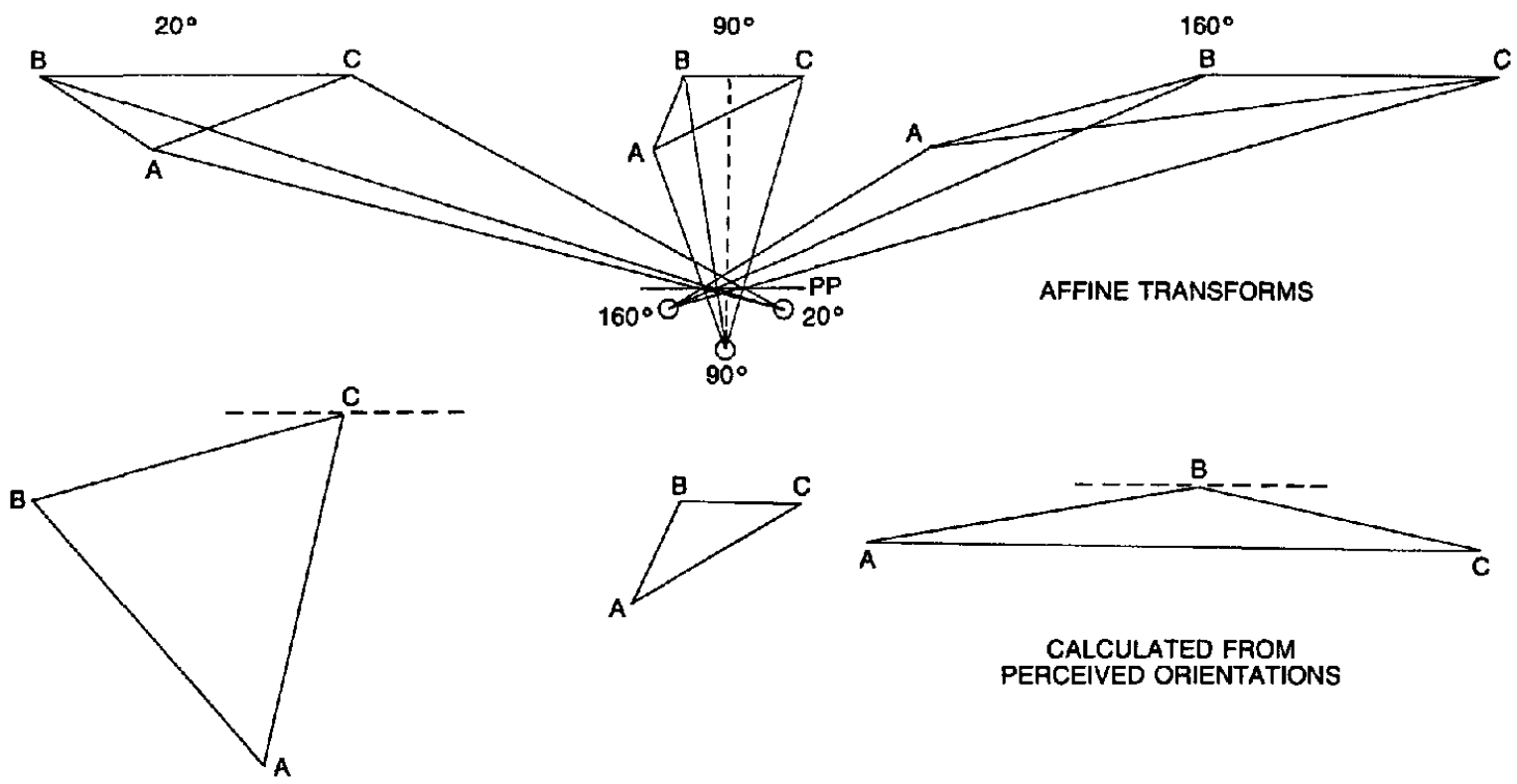

Figure 1. Top, center: Layout of the triangular array used in Goldstein (1987), as corrected by Cutting (1988), for optical compression by the camera lens. Top, left: Affine transformed array for a viewing angle of $20^{\circ}$. Top, right: Affine transformed array for a viewing angle of $160^{\circ}$. (The procedure used to construct this diagram is taken from Figure 3.1 of Cutting, 1986. The orientations shown in this diagram approximately match Cutting's calculated orientations. Deviations from Cutting's calculated orientations are most likely due to differences in assumed viewing positions. PP = picture plane; open circles $=$ positions of observers at viewing angles of $20^{\circ}, 90^{\circ}$, and $160^{\circ}$ relative to the picture plane. The dashed vertical line is the observer's line of sight for the $90^{\circ}$ viewing angle.) Bottom: Layouts calculated from the empirically determined perceived orientations in Figure 3 of Goldstein (1987; replotted in Figure 3 of Cutting, 1988). (Because perceived orientations do not provide information regarding size, the sizes of these triangles were determined by setting the length of side $\mathrm{BC}$ equal to the length of side $\mathrm{BC}$ of the corresponding triangle above. The triangles were constructed by drawing each line so that its orientation matches its empirically determined perceived orientation relative to the picture plane. The orientation of the picture planes for the lower triangles are indicated by dashed lines for the $20^{\circ}$ and $160^{\circ}$ viewing angles. The picture plane is omitted for the $90^{\circ}$ viewing angle for clarity, because the angle between $\mathrm{BC}$ and the picture plane is $2^{\circ}$.)

at the $20^{\circ}$ viewing angle. This difference reflects the large deviations between the predicted and observed functions in Cutting's Figure 3.

I conclude from these comparisons that in predicting less rotation for oblique stimulus orientations than for straight-on orientations, Cutting has demonstrated that the geometrical changes that occur with changes in viewing angle are at least partially responsible for the differential rotation effect. The differences between the predicted and empirical results, however, suggest that perceived orientation is also influenced by other factors. One possible factor, which is mentioned in Cutting's observation, is the visibility of the picture plane. $\mathrm{My}$ Experiment 5 (Goldstein, 1987) shows that eliminating the subjects' awareness of the picture plane, by having them view a back-lit transparency in the dark, causes large changes in the perceived orientation of a stimulus similar to $\mathrm{BC}$. When viewing angle is changed by $150^{\circ}$, this stimulus, which Cutting's model predicts should rotate $0^{\circ}$, rotates $60^{\circ}$ when viewed in the light (picture plane visible) and $113^{\circ}$ when viewed in the dark (picture plane not visible). Both the large deviation from the model's prediction and the large difference between the rotation in the picture-plane-visible and the picture-planenot-visible conditions (even though stimulus geometry is the same in the two conditions) argue against a purely geometrical explanation of how perceived orientation changes with changes in viewing angle.

Although stimulus geometry may not completely explain how perceived orientation changes with changes in viewing angle, the evidence supporting some role for geometry raises a potential paradox: On one hand, Cutting's predicted orientations are derived from spatial layouts; on the other hand, perceived orientation and spatial layout are affected differently by changes in viewing angle. Whereas perceived orientation varies with changes in viewing angle, spatial layout remains relatively constant. When observers are asked to duplicate a picture's spatial layout at different viewing angles and distances, their responses change far less than is predicted by the large changes in geometry that occur with changes in viewing angle (Goldstein, 1979, 1987, in press). Thus, even though geometrical changes are not translated into changes 
in an observer's perception of spatial layout, some of the information from these geometrical changes can be retrieved in the form of perceived orientations.

Why does spatial layout remain relatively constant even though retrievable geometrical information is available to the viewer? The answer may involve a compensation process (Kubovy, 1986; cf. Pirenne, 1970; Rosinski et al., 1980) that does not operate for perceived orientation. Whatever the answer, it is clear that there are differences between the mechanisms that determine spatial layout and perceived orientation. These differences led Cutting to suggest that perceived orientation is not a derived percept, because it "stems directly from visual information" (p. 311), whereas spatial layout is a derived percept, because it remains relatively constant in the face of changing visual information.

Whether the situation is as clearcut as this remains to be determined, but Cutting's distinction provides a good starting point for future research on this problem and reinforces one of the major points made in my article (Goldstein, 1987)that it is important to clearly distinguish between the attributes of perceived orientation and spatial layout in future research on the perception of pictures.

\section{References}

Cutting, J. E. (1986). Perception with an eye for motion. Cambridge, MA: MIT Press.

Cutting, J. E. (1988). Affine distortions of pictorial space: Some predictions for Goldstein (1987) that LaGournerie (1859) may have made. Journal of Experimental Psychology: Human Perception and Performance, 14, 305-311.
Ellis, S. R., Smith, S., \& McGreevy, M. A. (in press). Distortions of perceived visual directions out of pictures. Perception \& Psychophysics.

Goldstein, E. B. (1979). Rotation of objects in pictures viewed at an angle: Evidence for different properties of two types of pictorial space. Journal of Experimental Psychology: Human Perception and Performance, 5, 78-87.

Goldstein, E. B. (1987). Spatial layout, orientation relative to the observer, and perceived projection in pictures viewed at an angle. Journal of Experimental Psychology: Human Perception and Performance, 13, 256-266.

Goldstein, E. B. (in press). Perceived orientation, spatial layout and the geometry of pictures. In S. R. Ellis (Ed.), Proceedings of the Asilomar Conference on Spatial Displays and Spatial Instruments. Hillsdale, NJ: Erlbaum.

Kubovy, M. (1986). The psychology of perspective and Renaissance art. Cambridge, England: Cambridge University Press.

La Gournerie, J. de (1859). Traite de perspective linéaire contenant les trancés pour les tableaux plans et courbes, les bas-reliefs et les décorations théatrales, avec une théorie des effets de perspective [Treatise on linear perspective containing drawings for paintings, architectural plans and graphs, bas-reliefs and theatrical set design; with a theory of the effects of perspective]. Paris: Dalmont et Dunod.

Pirenne, M. H. (1970). Optics, painting \& photography. Cambridge, England: Cambridge University Press.

Rosinski, R. R., Mulholland, T., Degelman, D., \& Farber, J. (1980). Picture perception: An analysis of visual compensation. Perception \& Psychophysics, 28, 521-526.

Received August 3, 1987

Revision received August 10, 1987

Accepted August 10, 1987

\section{Call for Nominations for Editor of Behavioral Neuroscience}

The Publications and Communications Board has opened nominations for the editorship of Behavioral Neuroscience for the years 1990-1995. Richard F. Thompson is the incumbent editor. Candidates must be members of APA and should be available to start receiving manuscripts in early 1989 to prepare for issues published in 1990. Please note that the P\&C Board encourages more participation by women and ethnic minority men and women in the publication process and would particularly welcome such nominees. Submit nominations no later than August 1, 1988 to

$$
\begin{gathered}
\text { Martha Storandt } \\
\text { Department of Psychology } \\
\text { Washington University } \\
\text { St. Louis, Missouri } 63130 .
\end{gathered}
$$

Other members of the search committee are Byron Campbell, Mortimer Mishkin, Mark Rosenzweig, and Shepard Siegel. 